\title{
Translator's Acknowledgments
}

I am grateful to Professor William H. Jansen, who put my translation into English and whose sense of humor is obviously unshakeable. I would also like to offer thanks to Dr. Richard Dorson, who first suggested Krohn's work to me for translation, and Roger Abrahams, who encouraged me to submit my translation to the American Folklore Society's Bibliographic and Special Series. 
THIS PAGE INTENTIONALLY LEFT BLANK 